program being tailored and optimized to cater for space and time constraints. One obvious use of a course calculation program would be to make it continuous, using data derived from the navigation sub-system, with the output controlling the auto-helm. Such a program must be constructed from the start to allow for upward compatibility. It is also unlikely that a full U.S.A.S.I. Fortran compiler would be available, so Mr. Turner's logical If statements would be igNored. It may well be that the program would have to be written in Assembly language.

The message is clear.

(a) That a rhumb-line calculation may use Sadler's formulae 3 can be accepted.

(b) That the navigator will 'compute his tracks rapidly and accurately with a program such as this', is to be doubted.

To be constructive, it is suggested that any contributor to the Journal should polish his programs as he polishes his prose because many more people are in a position today to judge the result. Very few contributors would submit an article which contained a mathematical proof twice as long as it need be, so there seems to be little reason for including an inefficient program-it can always be omitted.

One last observation. If a G.P. computer becomes available, given the exception of avoiding a storm centre, what navigator in his right mind would use anything other than a great, or composite great, circle sailing?

\title{
REFERENCES
}

1 Turner, R. J. (1970). Rhumb-line sailing with a computer. This Journal, $23,233$.

2 Moss, B. J. (1969). Exploration with a computer on rhumb-line sailing. This Journal, $22,242$.

3 Sadler, D. H. (1956). Spheroidal sailing and middle latitude. This Journal, 9, 37 I.

\section{Application of Omega to Aircraft Navigation and Traffic Control}

\author{
E. R. Swanson \\ (Naval Electronics Laboratory Center, San Diego, California 92152)
}

THE many references to Omega in your excellent discussions on Navigation and traffic control published in the January issue were particularly gratifying to one who has worked on Omega for the past decade. Especially memorable was $\mathrm{Mr}$. Stringer's statement that '[Omega] satisfies the three $R$ 's-reliability, redundancy and range' and Mr. Jones' observation '. . . [Omega] is already the most widely deployed ground-based navigation aid in the world by a very substantial 
margin'. However, many contributors used words such as 'somewhat unreliable' and 'subject to real problems' when discussing ambiguity resolution. Perhaps some further remarks on ambiguity resolution are in order.

It is certainly true that ambiguity resolution is inherently a problem in Omega and, for that matter, in many other systems. Fundamentally, ambiguity resolution requires choosing between alternative possibilities, and as such there is always some inherent possibility of choosing incorrectly. The problem is not the conceptual possibility of error, but whether the probability is acceptable. It is thus necessary to examine the overall navigation problem before discussing the ambiguity problem specifically.

A recent study shows that Omega fix accuracy in North America, when using present skywave corrections, is about one mile, including the effects of sudden ionospheric disturbances and polar cap absorptions. Clearly, accuracy is not a factor even if $30-$ mile traffic separation is considered. Indeed, sufficient accuracy for this separation may be obtained by using the $3.4 \mathrm{kHz}$ difference frequency with an attendant lessening of the ambiguity resolution problem.

Reliability is crucial. If signals are received continuously and the equipment operates properly, then cycles are continuously tracked and an ambiguity problem would not arise. Present transmitting stations maintain a reliability of better than 99 per cent of scheduled transmission time. With redundant equipment now being constructed, reliability and availability should further improve. Considering redundant lines-of-position, the probability of simultaneous loss of sufficient transmissions to stop navigation should be negligibly small. Further, the better Omega receivers have been electronically reliable. It is quite possible that even a relatively sophisticated aircraft receiver will have an M.T.B.F. an order of magnitude better than the current M.T.B.F.'s cited by Mr. White. The primary cause of failure to track signals continuously will thus be associated with the possibility of extraordinarily high local noise levels such as the 'precipitation static' sometimes resulting from tribo-electric charging of an aircraft. Adequate statistics on the probability, intensity and duration of precipitation static seem unavailable. Signal loss for periods of 5 to $10 \mathrm{~min}$. has been observed on a number of flights. However, having personally logged about $100 \mathrm{hr}$. of flight time looking for precipitation static with notable lack of success, the problem must not be too severe, at least if proper loop antennas are employed.

Poor conditions of radio reception do not necessarily imply failure of Omega to track. In a recent transatlantic flight, Loran $A$, Loran $C$ and radio communications using standard antenna systems were lost for over $3 \mathrm{hr}$. while Omega was received continuously on a loop antenna. Nevertheless, Omega signals can occasionally be lost. If the loss prevails for a sufficient period, the aircraft D.R. will have developed sufficient uncertainty to make the carrier cycle uncertain. It is under these conditions, and only these, that Omega's internal capability for ambiguity resolution should be considered.

Loss of carrier cycle identification does not impose an inflexible requirement for ambiguity resolution. An alternative is available by navigating with one of the beat frequencies. In particular, the $3.4 \mathrm{kHz}$ can be used as a primary navigation variate to a fix accuracy of about 2 miles. Under extreme conditions, it is certainly far better to navigate on $3.4 \mathrm{kHz}$ with a possible error of 4 miles rather than force a choice between $10.2 \mathrm{kHz}$ cycles and risk an error of 8 miles. A satisfactory compromise is available. Ambiguities may be selectively resolved. The difference frequencies may be used for primary navigation until difference 
frequency fixes and carrier fixes are in 'close' agreement. Since the designer is free to define 'close' and since the probability of a discrepancy as great as a full carrier cycle between carrier and difference frequencies is nearly vanishingly small, it is possible to reduce the probability of an incorrect ambiguity resolution to an almost arbitrarily small figure. If, for example, the nominal probability of correct ambiguity resolution is 0.98 and a cycle match within $\frac{1}{3}$ cycle is demanded then the duty cycle for correct resolution will be reduced by about 10 per cent while the probability of misidentification will be reduced by a factor of 10 .

The above arguments may be facetiously summarized as follows. Considering improvements in aircraft loop antennas and wicks together with increased station power and improvements in information synthesis, we may postulate a probability of 0.1 for failure to track signals continuously on a transatlantic flight. Using the selective ambiguity probabilities given above we then conclude that a pilot flying $80 \mathrm{hr}$. per month in transatlantic service could expect: one failure per month to track continuously, but the lane ambiguity would be immediately resolved; one failure per year, but position would be obtained by extended operation on a difference frequency; and about one incorrect cycle identification in a 40-year career of flying the Atlantic. Even projected electronics M.T.B.F.'s will not support such Utopian performance nor is the preceding calculation valid at such an extreme probability. Nevertheless, although much of the input may have been estimated with considerable uncertainty, the estimates are probably not unrealistic and the result certainly would support $\mathrm{Mr}$. Jones' observation: 'Omega is not half so unreliable as some of its competitors!'

It may discourage some that the actual performance of Omega in aircraft cannot be specified exactly. However, this is not surprising. It has not been necessary to determine precisely how well Omega will operate; only that it will operate sufficiently well to justify implementation and adoption. Further, improvements can be expected in transmitting stations (number, power and reliability) and in aircraft receiving systems (wicks, antennas, receivers and information synthesis). Only propagation will not improve. However, propagation statistics support ambiguity resolution over 99 per cent of the time. Actual ambiguity resolution will also depend on prediction errors, but as knowledge of propagation prediction improves, errors will be reduced. Further, optimum information processing can improve ambiguity resolution. For example, with redundant signals available, it would usually be possible to restrict ambiguity resolution to those favourable signals which are not undergoing diurnal transition. In these conditions, the realizable probability of lane resolution should become about 0.98 or better. It is noteworthy too that the effects of modal interference as investigated so extensively by $\mathrm{Mr}$. Stringer, Dr. Burgess and their colleagues would not enter the problem as a random prediction error, but rather as an understood and bounded error. Bounded errors contribute to relatively few failures of ambiguity resolution. Using selective resolution, there would be a tendency to operate in a difference frequency mode until an aircraft had moved to a location where the required tolerances were achieved, at which time the ambiguity would be resolved correctly.

More important to overall performance than ambiguity resolution itself is the probability of maintaining continuous track on the carrier frequencies. As noted, tracking ability is very hard to assess, depending as it does on the occurrence and duration of extraordinarily high noise, the quality of the D.R. information available, and specific implementation details. However, the number of flights 
conducted with continuous tracking is certainly encouraging despite the use of relative crude D.R. information and low transmitted power. Quantitative data must await extensive flight operations with operational aircraft and equipment.

Flight data with an operational type of airborne receiver should soon be available. The Omega aircraft receiver built by Northrop and described by Daniel is now undergoing flight tests. (U.S. Institute of Navigation Journal, Winter 69-70 issue.) Older receivers, such as those used by R.A.E., N.R.L., and others (see for example articles by Sage and Enge in the same issue), can best be described as research or developmental models. The new Northrop receiver is intended as a prototype advanced engineering model for operational receivers and differs from others in that it includes the following features: self synchronization; internal computation of skywave corrections; redundant phase tracking of 10.2 , I $1 \frac{1}{3}$, and $13.6 \mathrm{kHz}$ from all stations, and readout in latitude and longitude or distance to go and lateral track error. The receiver is electronically simple, employing a minimum of radio-frequency circuitry and an 8192 r6-bit word computer which performs the phase tracking and various computing functions. It will easily interface with gyro, air speed, inertial, doppler or other aids that may be available. It should have an excellent capability for maintaining continuous position but, in the event of prolonged signal loss, will automatically shift to operate in a modified beat frequency mode and then perform selective ambiguity resolution when it can be confidently executed. Although many of the decision criteria within the receiver can benefit from continued refinement, it seems likely that operational trials will demonstrate a navigational capability significantly in excess of any now available. 